\title{
Modified Estimate of Subject Specific Index of Relative Performance in a Population
}

\author{
${ }^{1}$ Matthew Chukwuma Michael, ${ }^{2}$ Oyeka, I. C. A. and ${ }^{3}$ Bright Ajibade. \\ ${ }^{I}$ Department of Maths and Statistics, Delta State Polytechnics, P. M. B. 1030, Ogwashi-Uku, Delta State, \\ Nigeria. \\ ${ }^{2}$ Department of Statistics Nnamdi Azikiwe University, Awka, Anambra State, Nigeria. \\ ${ }^{3}$ Department of General Studies, Petroleum Training Institute, P.M.B 20, Effurun, Warri. Delta State. Nigeria.
}

\begin{abstract}
This paper proposes to develop the statistical method or measure for rank-ordering subjects relative to their performance or scores in a contest, test or condition in comparison with one another to enable guide decisions on preferential selection when opportunities or resources are scarce or limited.The methods which also provide appropriate modifications for their use when the population of research interest are numerical measurements, propose measures termed 'subject specific indices of relative performance' or 'subject specific relative performance indices' that are individual subject-specific rather loosely and globally-targeted, merely summary indices or averages. The proposed method using 'subject-specific relative performance indices' enables one easily and quickly estimate the median and other tiles of the distribution of the population.Test statistics based on the proposed indices are provided for testing desired hypotheses on patterns of relationship between performances or scores by subjects as well as about any percentiles of the population. The proposed methods are illustrated with some sample data and the method modified for use when the sampled population is numerical is shown to be relatively more powerful than the more generalized method used with measurements on at least the ordinal scale.
\end{abstract}

Key Words and Phrases: One Sample Data, Measures of Central Tendency, Measures of Dispersion, One Sample T-test, Sign Test, Median Test, Relative Relationships, Performance or scores in a contest, Preferential Selection, Rank-order, Index and Decision.

\section{Introduction}

In statistical analysis of one sample data a lot of attention has often been paid and devoted to measures of central tendency and measures of dispersion for these data sets, their estimation and hypothesis tests concerning them. If these are the only interest of a researcher, then the researcher can use any of the familiar statistical methods for the analysis of one-sample data such as the one-sample t-test, the sign tests, the median test or any other such methods to analyze the data (Arua et al 1997; Gibbons 1971; Oyeka 2012; Oyeka 2009, Oyeka 1996). But one-sample data sets intrinsically contain much more unexplored information than only a few parameters.

Such types of information include the relative relationships between the observations themselves. For example often assessors, decision makers, judges, teachers, etc may assess or judge a sample of subjects drawn from a population of subjects, objects or entities and score them on the basis of their performance or scores in a contest, test or condition for preferential selection relative to one another to fill vacant positions when opportunities or resources are limited or scarce. A medical health researcher or health management official may have data or information by some demographic classifications on subjects or patients on their state of health, medical test results, level of concentration of some contaminants, disease load, injury levels or other such conditions and may wish to relatively rank-order the subjects by the severity of their conditions by the various demographic classifications to guide decisions on the distribution and use of amenities when supplies are limited. In business, industry or in governmental affairs one may wish to know how various outfits, producers, suppliers and distributors of goods and services such as banks, transport operators, ministries, parastatals, etc compare in performance when juxtaposed against one another to guide any interventionist remedial actions by management or supervising body.

The problem before the decision makers is how using these observations to rationally select the required number of subjects, objects or outfits from the group of available subjectsor options to ensure that meritocracy is upheld in the presence of scarcity. Here although any desired hypothesis may be tested, this may not however be as important and useful as the need to find appropriate ways to systematically rank-order the subjects or available options according to their level of need or performance in a given test or situation to facilitate judicious selection to achieve a desired objective. This is because although hypothesis testing is important and useful, it may often not be as important and useful as the need to find ways to rank-order subjects 
or objects relative to one another for preferential selection of subjects from the sampled population of subjects or observations.

Hence there is a need to develop a method based on 'subject specific relative performance indices that enable one rank-order subjects relative to one another according to their performance or score in a contest or test for possible preferential selection. Also, such relative performance indices need to be used with data on at least the ordinal scale and reflect the direction and the relative magnitude of the observations. This paper proposes a generalized method of estimating subject specific indices of relative performance that may be used both when the observations are measurements on the ordinal scale and also when they are numerical information requiring the factoring-in of their relative magnitudes in their formulation.

Appropriate statistics based on the proposed specific relative performance indices both when the observations are non-numeric and when they are numeric are presented for comparing patterns of relationships between performance or score s by subjects as well as for testing any desired hypotheses about the various percentiles of the sampled population.

\section{The Proposed Method}

Let $x_{i}$ be the observation, value or outcome of a condition, event or experiment for $i=1,2, \ldots, n$. The observations should be measurements on at least the ordinal scale but may or may not be continuous or even numeric.

To develop the proposed method for the general case in which the sampled population is a measurement on only the ordinal scale that is not necessarily numeric we may let

For $i, j=1,2, \ldots, n ; i \neq j$

$$
U_{i j}=\left\{\begin{array}{c}
1 \text { if } x_{i} \text { isahigher (better, more) scorethan } x_{j} \\
0 \text { if } x_{i} \text { isthesamescoreas }(\text { equalto }) x_{j} \\
-1, \text { if } x_{i} \text { isalower }(\text { worse, less }) \text { scorethan } x_{j}
\end{array}\right.
$$

Let

where

$$
\pi_{i}^{+}=P\left(U_{i j}=1\right) ; \pi_{i}^{0}=P\left(U_{i j}=0\right) ; \pi_{i}^{-}=P\left(U_{i j}=-1\right)
$$

Let

$$
\pi_{i}^{+}+\pi_{i}^{0}+\pi_{i}^{-}=1
$$

Now

$$
W_{i}=\sum_{\substack{j=1 \\ j \neq i}}^{n} U_{i j}
$$

Also

$$
E\left(U_{i j}\right)=\pi_{i}^{+}-\pi_{i}^{-} ; \operatorname{Var}\left(U_{i j}\right)=\pi_{i}^{+}+\pi_{i}^{-}-\left(\pi_{i}^{+}-\pi_{i}^{-}\right)^{2}
$$

$$
E\left(W_{i}\right)=\sum_{\substack{j=1 \\ j \neq i}}^{n} E\left(U_{i j}\right)=(n-1)\left(\pi_{i}^{+}-\pi_{i}^{-}\right)
$$

And

$$
\operatorname{Var}\left(W_{i}\right)=\sum_{\substack{j=1 \\ j \neq i}}^{n} \operatorname{Var}\left(U_{i j}\right)=(n-1)\left(\pi_{i}{ }^{+}-\pi_{i}{ }^{-}-\left(\pi_{i}{ }^{+}-\pi_{i}{ }^{-}\right)^{2}\right)
$$

Now $\pi_{i}{ }^{+}, \pi_{i}{ }^{0}$ and $\pi_{i}{ }^{-}$are respectively the probabilities that the score, outcome or condition of the $i^{t h}$ subject in time or space is higher (better, more) than the scores, outcomes or conditions of all other subjects in time or space from the sampled population. Their sample estimates are respectively

$$
{\hat{\pi}_{i}}^{+}=\frac{f_{i}^{+}}{n-1} ; \hat{\pi}_{i}^{0}=\frac{f_{i}^{0}}{n-1} ; \hat{\pi}_{i}^{-}=\frac{f_{i}^{-}}{n-1} \ldots
$$

For $i=1,2, \ldots, n$ where $f_{i}^{+}, f_{i}^{0}$ and $f_{i}^{-}$are respectively the number of times or frequency by which (by how much) the $i^{\text {th }}$ subject's performance, outcome or condition in time or space is higher (better, more), the same as (equal to) or lower (worse, less) than the performance, outcomes or conditions of all other subjects in the sample. In other words $f_{i}^{+}, f_{i}^{0}$ and $f_{i}^{-}$are respectively the number of $1 \mathrm{~s}, 0 \mathrm{~s}$ and $-1 \mathrm{~s}$ in the frequency distribution of the $n-1$ values of these numbers in $U_{i j}$, for $i, j=1,2, \ldots, n ; j \neq i$

The difference or gap between $f_{i}^{+}$and $f_{i}^{-}$namely

$$
W_{i}=f_{i}^{+}-f_{i}^{-}=(n-1)\left(\hat{\pi}_{i}^{+}-\hat{\pi}_{i}^{-}\right)
$$

which measure by how much the perform, outcome or condition of the $i^{\text {th }}$ subject is more serious, higher (better, greater) less by how much that subjects performance, outcome or condition is less serious, lower (worse, 
smaller) than the conditions or outcomes of all other subjects in the sampled population. Thus $W_{i}$ may be referred to as subject specific index of relative performance or subject specific relative performance measuring the frequency or by how much the performance, outcome or condition of the $i^{\text {th }}$ subject in time or space is higher (or lower) than that is in comparison or relative to the performance, outcome or conditions of all other subjects in the population.

If the performance or outcome of the $i^{t h}$ subject is higher (better, more) than those of all other subjects from the sampled population and there are no ties in $W_{i}$, then $W_{i}=f_{i}^{+}=n-1 ; f_{i}^{-}=0 ; \hat{\pi}_{i}^{+}=1 ; \hat{\pi}_{i}^{-}=0$ and the $i^{t h}$ subject would be considered and ranked the highest, best achiever or performer. If the $i^{t h}$ subject scores lower than all other subjects in the sample then $W_{i}=f_{i}^{-}=-(n-1) ; f_{i}^{+}=0 ;{\pi_{i}}^{+}=0 ; \hat{\pi}_{i}^{-}=1$ and the $i^{\text {th }}$ subject would be ranked last as the lowest performer or achiever and hence the worst of all the subjects. Hence rank-ordering the values of $W_{i}$ from the highest or largest to the lowest or smallest or vice-versa, assigning the largest $W_{i}$ the rank 1 (or $n$ ) the next largest the rank 2 (or $n-1$ ) and so on until the smallest is assigned the rank 1 (or $n$ ) enables one arrange the subjects for preferential selection according to their performance, score, outcome or condition in a test or experiment. Tied values of $W_{i}$ are assigned their mean ranks.

Increasingly large and positive values of $W_{i}$ would indicate that the $i^{\text {th }}$ subject's condition, outcome or performance is not serious; that is, good (serious, bad); while increasingly small and negative values of $W_{i}$ would indicate the converse, that is that the $i^{\text {th }}$ subject performance, outcome or condition is serious; that is bad(not serious, good) compared with all other subjects given the test or experiment of interest.

If the $i^{t h}$ subject's performance, outcome or condition is higher (better, more) than those of one-half of the other subjects but less than those of another one-half and there are no tied observations and hence no ties in $W_{i}$, then $W_{i}=0, \hat{\pi}_{i}^{+}=\hat{\pi}_{i}^{-}$if $n$ is odd and hence assigned the median rank; or the two middle-most values of $W_{i}=$ $0, \hat{\pi}_{i}{ }^{+}=\hat{\pi}_{i}{ }^{-}$if $n$ is odd and hence assigned the median rank; or the two middle-most values of $W_{i}$ are 1 and -1 respectively summing to $n$ if $n$ is even so that the average of their ranks is the median rank. In these cases the median of the sample and hence an estimate of the population median is the value of the observation or outcome $x_{i}$ whose $W_{i}$ is zero if $n$ is odd or the average of the two outcomes with $W_{i}$ values of 1 and -1 respectively also summing to zero, if $n$ is even. In general however the sample median and hence the sample estimate of the population median whether or not there are tied observations is the value of the observation with the middlemost ranked $W_{i}$ if $n$ is odd or the average of the two observations with the two middle-most ranked values of $W_{i}$ if $n$ is even which are assigned the median rank in the rank ordering of the $W_{i}$ values or indices for $i=$ $1,2, \ldots, n$.

As already noted, the sample variance of $W_{i}$ is from equation 7 ;

$$
\operatorname{Var}\left(W_{i}\right)=(n-1)\left(\hat{\pi}_{i}^{+}+{\hat{\pi}_{i}}^{-}-\left(\hat{\pi}_{i}^{+}-\hat{\pi}_{i}^{-}\right)^{2}\right)
$$

If the $i^{\text {th }}$ subject's condition is better (or worse) than those of one-half of the subjects and also worse (or better) than those of another one-half of the sampled population then one would expect that $E\left(W_{i}\right)=(n-1)\left(\pi_{i}^{+}-\right.$ $\pi i^{-}=0$, so that $\pi i+-\pi i^{-}=0$. However a more general null hypothesis would be that the $i t h$ subjects specific relative performance index is at least some specified value $W_{i 0}$ say; that is the null hypothesis of interest may be

$$
H_{0}: E\left(W_{i}\right) \geq W_{i 0} \text { versus }_{1}: E\left(W_{i}\right)<W_{i 0}(\text { say })
$$

The null hypothesis of equation 11 be tested using the test statistic,

$$
\chi^{2}=\frac{\left(W_{i}-W_{i 0}\right)^{2}}{\operatorname{Var}\left(W_{i}\right)}=\frac{\left((n-1)\left(\left(\hat{\pi}_{i}^{+}-\hat{\pi}_{i}^{-}\right)-W_{i 0}\right)\right)^{2}}{(n-1)\left(\hat{\pi}_{i}^{+}+\hat{\pi}_{i}^{-}-\left(\hat{\pi}_{i}^{+}-\hat{\pi}_{i}^{-}\right)^{2}\right)} \ldots \ldots \ldots \ldots
$$

for $i=1,2, \ldots, n$; which under $H_{0}$ has approximately the chi-square distribution with 1 degree of freedom for sufficiently large $n$.

The null hypothesis of equation 11 is rejected at the $\alpha$-level of significance if

otherwise $H_{0}$ is accepted.

$$
\chi^{2} \geq \chi_{1-\alpha ; 1}^{2}
$$

It is possible that a decision is to be taken to remediate, that is intervene with the $i^{\text {th }}$ subject's condition only if that subject's specific relative performance index $W_{i}$ is statistically less than $W_{i 0}$ at a specified $\alpha-$ level. In such a situation and following equation 13, for the subject to qualify for a remedial action the subject's specific relative performance index $W_{i}$ must satisfy the equation.

$$
\begin{aligned}
W_{i}=\sqrt{(n-1)\left(\hat{\pi}_{i}^{+}+\hat{\pi}_{i}^{-}-\left(\hat{\pi}_{i}^{+}-\hat{\pi}_{i}^{-}\right)^{2}\right)} \chi_{1-\alpha ; 1}^{2} \leq W_{i} \\
\leq W_{i}+\sqrt{(n-1)\left(\hat{\pi}_{i}^{+}+\pi_{i}^{-}-\left(\hat{\pi}_{i}^{+}-\hat{\pi}_{i}^{-}\right)^{2}\right) \chi_{1-\alpha ; 1}^{2}}
\end{aligned}
$$


To determination whether the $i^{t h}$ and $j^{t h}$ subject's time periods or locations differ in their specific relative performance indices, we may let $W_{i j}$ be the sample estimate of the difference between the specific performance indices of the subjects expressed as

$$
W_{i j}=W_{i}-W_{j}=(n-1)\left(\left(\hat{\pi}_{i}^{+}-\hat{\pi}_{i}^{-}\right)-\left(\hat{\pi}_{j}^{+}-\hat{\pi}_{j}^{-}\right)\right)
$$

with corresponding variance

where

$$
\operatorname{Var}\left(W_{i j}\right)=\operatorname{Var}\left(W_{i}-W_{j}\right)=\operatorname{Var}\left(W_{i}\right)+\operatorname{Var}\left(W_{j}\right)-2 \operatorname{Cov}\left(W_{i} ; W_{j}\right)
$$

$$
\operatorname{Cov}\left(W_{i}, W_{j}\right)=E\left(W_{i} \cdot W_{j}\right)-E\left(W_{i}\right) \cdot E\left(W_{j}\right)=E\left(W_{i} \cdot W_{j}\right)-(n-1)^{2}\left(\pi_{i}^{+}-\pi_{i}^{-}\right)\left(\pi_{j}^{+}-^{-} \pi_{j}^{-}\right)
$$

Now $E\left(W_{i} \cdot W_{j}\right)=\sum_{\substack{l=1 \\ l=i}}^{n} \sum_{\substack{h=1 \\ h=j}}^{n} E\left(U_{i l} . U_{j h}\right)$, where $U_{i l} . U_{j h}$ can assume only the values 1,0 and -1 . It assumes the value 1 or the value -1 with probability $\pi_{i}{ }^{+}-\pi_{j}{ }^{+}+\pi_{i}{ }^{-}-\pi_{j}{ }^{-}$; It assumes the value 0 if and only if $U_{i l}$ and $U_{j h}$ both assume the value 0 or $U_{i l}$ assume the value 0 no matter the value assumed by $U_{i l}$ or $U_{j h}$ assumes the 0 no matter the values assumed by $U_{i l}$ with probability $\pi_{i}{ }^{0} \cdot \pi_{j}{ }^{0}+\pi_{i}{ }^{0}\left(\pi_{j}{ }^{+}+\pi_{j}{ }^{-}\right)+\pi_{j}{ }^{0}\left(\pi_{i}{ }^{+}+\pi_{i}{ }^{-}\right)$; and the value 1 if and only if $U_{i l}$ assumes he value 1 and $U_{j h}$ assumes the value -1 or $U_{j h}$ assumes the value 1 and $U_{i l}$ assumes the value -1 with probability $\pi_{i}{ }^{+} . \pi_{j}{ }^{-}+\pi_{i}{ }^{-} \cdot \pi_{j}{ }^{+}$. Collecting terms we have that

$$
\operatorname{Cov}\left(W_{i}, W_{j}\right)=(n-1)^{2}\left(\left(\pi_{i}^{+} \cdot \pi_{j}^{+}+\pi_{i}^{-} \cdot \pi_{j}^{-}-\left(\pi_{i}^{+} \cdot \pi_{j}^{-}+\pi_{i}^{-} \cdot \pi_{j}^{+}\right)\right)-\left(\pi_{i}^{+}-\pi_{i}^{-}\right)\left(\pi_{j}^{+}-\pi_{j}^{-}\right)\right)=0
$$

Hence using equation 10 we have

$$
\begin{gathered}
\operatorname{Var}\left(W_{i j}\right)=\operatorname{Var}\left(W_{i}\right)+\operatorname{Var}\left(W_{j}\right) \\
=(n-1)\left(\left({\hat{\pi}_{i}}^{+}+\hat{\pi}_{i}^{-}-\left(\hat{\pi}_{i}^{+}-\hat{\pi}_{i}^{-}\right)^{2}\right)+\left(\hat{\pi}_{j}^{+}+\hat{\pi}_{j}^{-}-\left(\hat{\pi}_{j}^{+}-\hat{\pi}_{j}{ }^{-}\right)^{2}\right)\right) \ldots
\end{gathered}
$$

Therefore to test the null hypothesis that subjects $i$ and $j$ do not differ in their specific relative performance indices we may use the test statistic

$$
\begin{aligned}
\chi^{2}=\frac{W_{i j}^{2}}{\operatorname{Var}\left(W_{i j}\right)}= & \frac{\left(W_{i}-W_{j}\right)^{2}}{\operatorname{Var}\left(W_{i}\right)+\operatorname{Var}\left(W_{j}\right)} \\
& =\frac{(n-1)\left(\left(\hat{\pi}_{i}^{+}-\hat{\pi}_{i}^{-}\right)-\left(\hat{\pi}_{j}^{+}-\hat{\pi}_{j}^{-}\right)\right)^{2}}{\left(\hat{\pi}_{i}^{+}+\hat{\pi}_{i}^{-}-\left(\hat{\pi}_{i}^{+}-\hat{\pi}_{i}^{-}\right)^{2}\right)+\left(\hat{\pi}_{j}^{+}+\hat{\pi}_{j}^{-}-\left(\hat{\pi}_{j}^{+}-\hat{\pi}_{j}^{-}\right)^{2}\right)}
\end{aligned}
$$

for $i, j=1,2, \ldots, n ; i \neq j$; which has approximately the chi-square distribution with 1 degree of freedom for sufficiently large $n$. The null hypothesis is rejected at $\alpha$-level of significance if equation 13 is satisfied; otherwise the null hypothesis is accepted.

A sample estimate of the overall relative performance index for all subjects, time periods, or locations if desired is easily obtained as

whose sample variance is

$$
W=\sum_{i=1}^{n} W_{i}=(n-1) \sum_{i=1}^{n}\left(\hat{\pi}_{i}^{+}-\hat{\pi}_{i}^{-}\right)=\sum_{i=1}^{n}\left(f_{i}^{+}-f_{i}^{-}\right)
$$

$$
\operatorname{Var}(W)=(n-1) \sum_{i=1}^{n}\left(\hat{\pi}_{i}^{+}+\hat{\pi}_{i}^{-}-\left(\hat{\pi}_{i}^{+}-\hat{\pi}_{i}{ }^{-}\right)^{2}\right) \ldots
$$

To test the null hypothesis that all subjects equally perform well or that the overall performance index is zero, we may use the test statistic

$$
\chi^{2}=\frac{W^{2}}{\operatorname{Var}(W)}
$$

rejecting the null hypothesis if equation 13 is satisfied.

If the observations or scores $x_{i}$ are numeric then equation 1 may be modified to read

for $i, j=1,2, \ldots, n ; j \neq i$

$$
U_{i j}=\left\{\begin{array}{c}
t_{i j}{ }^{+}=x_{i}-x_{j}, \text { if } x_{i}>x_{j} \\
0, \text { if } x_{i}=x_{j} \\
t_{i j}{ }^{-}=-\left(x_{j}-x_{i}\right), \text { if } x_{i}<x_{j}
\end{array}\right.
$$

Equations 2-4 remain as defined.

Now

$$
E\left(U_{i j}\right)=t_{i j}{ }^{+} \cdot \pi_{i}{ }^{+}+t_{i j}{ }^{-} \cdot \pi_{i}{ }^{-} ; \operatorname{Var}\left(U_{i j}\right)=t_{i j}{ }^{+* 2} \cdot \pi_{i}{ }^{+}+t_{i j}{ }^{-* 2} \cdot \pi_{i}{ }^{-}-\left(t_{i j}{ }^{+} \cdot \pi_{i}{ }^{+}+t_{i j}{ }^{-} \cdot \pi_{i}{ }^{-}\right)^{2}
$$

From equation 4 we have that 


$$
E\left(W_{i}\right)=\sum_{\substack{j=1 \\ j \neq i}}^{n} E\left(U_{i j}\right)=\sum_{\substack{j=1 \\ j \neq i}}^{n}\left(t_{i j}{ }^{+} \cdot \pi_{i}^{+}+t_{i j}{ }^{-} \cdot \pi_{i}^{-}\right)\left(t_{i .}{ }^{+} \cdot \pi_{i}^{+}+t_{i .} \cdot \cdot \pi_{i}^{-}\right)
$$

where

$$
t_{i}^{+}=\sum_{\substack{j=1 \\ j \neq i}}^{n} t_{i j}{ }^{+} ; t_{i}^{-}=\sum_{\substack{j=1 \\ j \neq i}}^{n} t_{i j}^{-}
$$

Note that $t_{i}{ }^{+}$and $t_{i}^{-}$are respectively the total amount, scores or values by which the score $x_{i}$ by the $i^{\text {th }}$ subject is greater than and smaller than the scores by all the other subjects in the sampled population, for $i=1,2, \ldots, n$.

Also

$$
\begin{aligned}
& \operatorname{Var}\left(W_{i}\right)=\sum_{\substack{j=1 \\
j \neq i}}^{n} \operatorname{Var}\left(U_{i j}\right)=\sum_{\substack{j=1 \\
j \neq i}}^{n}\left(t_{i j}{ }^{+* 2} \pi_{i}{ }^{+}+t_{i j}{ }^{-* 2} \pi_{i}{ }^{-}-\left(t_{i j}{ }^{+} \cdot \pi_{i}{ }^{+}+t_{i j}{ }^{+} \cdot \pi_{i}{ }^{-}\right)^{2}\right) \\
= & \sum_{\substack{j=1 \\
j \neq i}}^{n}\left(t_{i j}{ }^{+* 2} \cdot \pi_{i}^{+}+t_{i j}{ }^{-* 2} \cdot \pi_{i}^{-}-\left(t_{i j}{ }^{+* 2} \cdot \pi_{i}^{+* 2}+t_{i j}{ }^{-* 2} \cdot \pi_{i}{ }^{-* 2}+2 t_{i j}{ }^{+} \cdot t_{i j}{ }^{-} \cdot \pi_{i}{ }^{+} \cdot \pi_{i}{ }^{-}\right)\right)
\end{aligned}
$$

Which when further simplified and evaluated yields

$$
\operatorname{Var}\left(W_{i}\right)=\pi_{i}^{+}\left(1-\pi_{i}^{+}\right) \sum_{\substack{j=1 \\ j \neq i}}^{n} t_{i j}^{+* 2}+\pi_{i}^{-}\left(1-\pi_{i}^{-}\right) \sum_{\substack{j=1 \\ j \neq i}}^{n} t_{i j}{ }^{-* 2}
$$

Since $t_{i j}{ }^{+} \cdot t_{i j}{ }^{-}=0$ for $j \neq i$;

The sample estimates of $W_{i}$ and its variance obtained using equation 8 in equations 23 and 25 are respectively

And

$$
W_{i}=t_{i .}{ }^{+} \hat{\pi}_{i}^{+}+t_{i .}^{-} \cdot \hat{\pi}_{i}^{-}
$$

$$
\operatorname{Var}\left(W_{i}\right)=\hat{\pi}_{i}^{+}\left(1-\hat{\pi}_{i}^{+}\right) \cdot \sum_{\substack{j=1 \\ j \neq i}}^{n} t_{i j}{ }^{+* 2}+\hat{\pi}_{i}{ }^{-}\left(1-\hat{\pi}_{i}{ }^{-}\right) \sum_{\substack{j=1 \\ j \neq i}}^{n} t_{i j}{ }^{-*}
$$

for $i=1,2, \ldots, n$.

With these modifications, analysis may now continue without any further problems for example the null hypothesis of equation 11 may still be tested using the test statistic of equation 12 but now replacing $W_{i}$ and its variance, $\operatorname{Var}\left(W_{i}\right)$, with the values obtained using equations 26 and 27 respectively. Other hypothesis may be similarly approximately tested.

Also to determine whether the $i^{\text {th }}$ and $j^{\text {th }}$ subjects differ in their performance we have that the counterpart of equation 15 when the observations are numeric is from equation 26

$$
W_{i j}=W_{i}-W_{j}=\left(t_{i}{ }^{+} \cdot \hat{\pi}_{i}^{+}+t_{i}{ }^{-} \hat{\pi}_{i}{ }^{-}\right)-\left(t_{j}{ }^{+} \hat{\pi}_{j}{ }^{+}+t_{j}{ }^{-} \cdot \hat{\pi}_{j}{ }^{-}\right)
$$

whose variance is easily shown to be

or equivalently using equation 27

$$
\operatorname{Var}\left(W_{i j}\right)=\operatorname{Var}\left(W_{i}-W_{j}\right)=\operatorname{Var}\left(W_{i}\right)+\operatorname{Var}\left(W_{j}\right)
$$

$$
\begin{aligned}
\operatorname{Var}\left(W_{i j}\right)=\operatorname{Var} & \left(W_{i}\right)+\operatorname{Var}\left(W_{j}\right) \\
& =\left({\hat{\pi}_{i}}^{+}\left(1-\hat{\pi}_{i}^{+}\right) \cdot \sum_{\substack{i=1 \\
l \neq i}}^{n} t_{i l}{ }^{+* 2}+\hat{\pi}_{i}^{-}\left(1-\hat{\pi}_{i}^{-}\right) \sum_{\substack{i=1 \\
l \neq i}}^{n} t_{i l}{ }^{-* 2}\right) \\
& +\left({\hat{\pi}_{j}}^{+}\left(1-\hat{\pi}_{j}{ }^{+}\right) \sum_{\substack{h=1 \\
h \neq j}}^{n} t_{j h}{ }^{+* 2}+\hat{\pi}_{j}^{-}\left(1-\hat{\pi}_{j}^{-}\right) \sum_{\substack{h=1 \\
h \neq j}}^{n} t_{j h}{ }^{-* 2}\right) \ldots \ldots . .(30
\end{aligned}
$$

for $i, j=1,2, \ldots, n ; i \neq j$

To show that equations 29 and 30 are valid it is only necessary to show that $\operatorname{Cov}\left(W_{i j}, W_{j}\right)=0$. To show this it is sufficient to show that $\operatorname{Cov}\left(U_{i l} ; U_{j h}\right)=0$.

Now $\quad \operatorname{Cov}\left(U_{i l} ; U_{j h}\right)=E\left(U_{i l} ; U_{j h}\right)-E\left(U_{i l}\right) * E\left(U_{j h}\right)=E\left(U_{i l} \cdot U_{j h}\right)-\left(t_{i l}{ }^{+} \cdot \pi_{i}{ }^{+}+t_{i l}{ }^{-} \cdot \pi_{i}{ }^{-}\right)\left(t_{j h}{ }^{+} \cdot \pi_{j}{ }^{+}+\right.$ $t j h-\pi j$ ..(31) 
Now $\left(U_{i l} \cdot U_{j h}\right)$ can assume only the values $t_{i l}{ }^{+} \cdot t_{j h}{ }^{+}, t_{i l}{ }^{+} \cdot t_{j h}{ }^{-} ; t_{i l}{ }^{-} \cdot t_{j h}{ }^{+}$and $t_{i l}{ }^{-} \cdot t_{j h}{ }^{-}$with probabilities $\pi_{i}^{+} . \pi_{j}^{+} ; \pi_{i}^{+} . \pi_{j}{ }^{-} ; \pi_{i}^{-} \cdot \pi_{j}^{+}$and $\pi_{i}^{-} \cdot \pi_{j}^{-}$. Hence,

$$
\begin{aligned}
\operatorname{Cov}\left(U_{i l} ; U_{j h}\right)= & \left(t_{i l}{ }^{+} \cdot t_{j h}{ }^{+} \cdot \pi_{i}{ }^{+} \pi_{j}{ }^{+}+t_{i l}{ }^{+} t_{j h}{ }^{-} \pi_{i}{ }^{+} \pi_{j}{ }^{-}+t_{i l}{ }^{-} t_{j h}{ }^{+} \pi_{i}{ }^{-} \pi_{j}{ }^{+}+t_{i l}{ }^{-} t_{j h}{ }^{-} \pi_{i}{ }^{-} \pi_{j}{ }^{-}\right) \\
& -\left(t_{i l}{ }^{+} \pi_{i}{ }^{+}+t_{i l}{ }^{-} \pi_{i}{ }^{-}\right)\left(t_{j h}{ }^{+} \pi_{j}{ }^{+}+t_{j h}{ }^{-} \pi_{j}{ }^{-}\right)=0
\end{aligned}
$$

Finally, the sample estimates of the overall relative performance index for all subjects, time periods or locations and the corresponding variance are obtained from equations 26 and 27 as respectively

And

$$
W=\sum_{i=1}^{n} W_{i}=\sum_{i=1}^{n}\left(t_{i}^{+} \hat{\pi}_{i}^{+}+t_{i}{ }^{-} \hat{\pi}_{i}^{-}\right)
$$

$$
\operatorname{Var}(W)=\sum_{i=1}^{n} \operatorname{Var}\left(W_{i}\right)=\sum_{i=1}^{n}\left(\hat{\pi}_{i}{ }^{+}\left(1-\hat{\pi}_{i}{ }^{+}\right) \cdot \sum_{\substack{j=1 \\ j \neq i}}^{n} t_{i j}{ }^{+* 2}+\hat{\pi}_{i}{ }^{-}\left(1-\hat{\pi}_{i}{ }^{+}\right) \cdot \sum_{\substack{j=1 \\ j \neq i}}^{n} t_{i j}{ }^{-* 2}\right) \ldots
$$

The null hypothesis that the overall relative performance index or value is zero may be tested using equation 20, replacing $W$ and its variance $\operatorname{Var}(W)$ with the values obtained using equation 31 and 32 respectively.

Now note that the rank assigned to each subject in the rank-ordering of subject relative performance indices for preferential selection whether based on equation 9 or equation 26 is the same rank that would have been assigned to that subject if the observations on these subjects were merely ranked in the usual way. However the proposed method enables easy and quick estimation with minimal calculation of the percentiles and other tiles of the distribution of the population of interest using their ranks. Thus, if $W_{i(r)}$ designates the relative performance index by the $i^{t h}$ subject assigned the rank $r$, then the $k^{t h}$ percentile of the relative performance indices is estimated as

$$
K_{p}=\left\{\begin{array}{c}
W_{i}\left(\frac{k(n+1)}{p}\right) \text { ifnisodd } \\
\frac{W_{i}\left(\frac{k \cdot n}{p}\right)+\left(W_{i}\left(\frac{k \cdot n}{p}\right)+1\right)}{2} \text { ifniseven }
\end{array}\right.
$$

For $k=1,2, \ldots p-1 ; p=100 . i=1,2, \ldots n$

The value of the observation $x_{i}$ or the average of the observations depending on whether $n$ is odd or even corresponding to these indices is then the same estimate of the $k^{\text {th }}$ percentile of the population.

\section{Illustrative Example}

We here illustrated the proposed method with the following data on cholesterol levels of a random sample of 11 male school teachers from a certain community.

\begin{tabular}{|c|c|c|c|c|c|c|c|c|c|c|c|}
\hline S/N & 1 & 2 & 3 & 4 & 5 & 6 & 7 & 8 & 9 & 10 & 11 \\
\hline $\begin{array}{c}\text { Cholesterol } \\
\text { level }\end{array}$ & 267 & 193 & 168 & 250 & 182 & 194 & 185 & 180 & 200 & 205 & 182 \\
\hline $\begin{array}{c}\text { Assigned } \\
\text { rank }\end{array}$ & 1 & 6 & 11 & 2 & 8.5 & 5 & 7 & 10 & 4 & 3 & 8.5 \\
\hline
\end{tabular}

Now the application of equation 1 and 21 to the above cholesterol data enables us obtain the values of $U_{i j}$ and hence the summary values $f_{i}^{+}, f_{i}{ }^{0}, f_{i}^{-}, W_{i}, t_{i}{ }^{+}, t_{i}{ }^{-}, r_{i}$ and other statistics presented in table. 
Table 1: Subject Specific Relative Performance Indices on the Cholesterol levels of a random sample of male school teachers

\begin{tabular}{|c|c|c|c|c|c|c|c|c|c|c|c|c|c|c|}
\hline & $\begin{array}{c}\text { Chol } \\
\text { Leve } \\
\text { l }\end{array}$ & $f_{i}^{+}$ & $f_{i}{ }^{0}$ & $f_{i}^{-}$ & $\widehat{\pi}_{i}{ }^{+}$ & $\widehat{\pi}_{i}{ }^{0}$ & $\widehat{\pi}_{i}^{-}$ & $\begin{array}{l}W_{i}(9)\left(f_{i}^{+}\right. \\
\left.-f_{i}^{-}\right)\end{array}$ & $r_{i}$ & $t_{i}^{+}$ & $t_{i}^{-}$ & $\widehat{\pi}_{i}^{+} t_{i}^{+}$ & $\widehat{\pi}_{i}^{-} t_{i}^{-}$ & $W_{i}(26)$ \\
\hline 1 & 267 & 10 & 0 & 0 & 1 & 0 & 0 & 10 & 1 & 731 & 0 & 731 & 0 & 731 \\
\hline 2 & 193 & 5 & 0 & 5 & $\begin{array}{l}0 . \\
5 \\
\end{array}$ & 0 & $\begin{array}{l}0 . \\
5\end{array}$ & 0 & 6 & 68 & 151 & 34 & -75.5 & -41.5 \\
\hline 3 & 168 & 0 & 0 & 10 & 0 & 0 & 1 & -10 & 11 & 358 & 0 & 0 & -358 & -358 \\
\hline 4 & 250 & 9 & 0 & 1 & $\begin{array}{l}0 . \\
9\end{array}$ & 0 & $\begin{array}{c}0 . \\
1\end{array}$ & 8 & 2 & 561 & 17 & 504.9 & -1.7 & 503.2 \\
\hline 5 & 182 & 2 & 1 & 7 & $\begin{array}{l}0 . \\
2\end{array}$ & $\begin{array}{l}0 . \\
1\end{array}$ & $\begin{array}{l}0 . \\
7\end{array}$ & -5 & $\begin{array}{l}8 . \\
5 \\
\end{array}$ & 16 & 220 & 3.2 & -154 & 150.8 \\
\hline 6 & 194 & 6 & 0 & 4 & $\begin{array}{c}0 . \\
6\end{array}$ & 0 & $\begin{array}{l}0 . \\
4\end{array}$ & 2 & 5 & 74 & 146 & 44.4 & -58.4 & -14 \\
\hline 7 & 185 & 4 & 0 & 6 & $\begin{array}{l}0 . \\
4\end{array}$ & 0 & $\begin{array}{c}0 . \\
6\end{array}$ & -2 & 7 & 28 & 200 & 11.2 & -120 & 108.8 \\
\hline 8 & 180 & 1 & 0 & 9 & $\begin{array}{c}0 . \\
1\end{array}$ & 0 & $\begin{array}{l}0 . \\
9\end{array}$ & -8 & 10 & 14 & 234 & 1.4 & $\begin{array}{c}- \\
210 . \\
6\end{array}$ & -209.2 \\
\hline 9 & 200 & 7 & 0 & 3 & $\begin{array}{l}0 . \\
7\end{array}$ & 0 & $\begin{array}{l}0 . \\
3\end{array}$ & 4 & 4 & 116 & 122 & 81.2 & $36 . .6$ & 44.6 \\
\hline 10 & 205 & 8 & 0 & 2 & $\begin{array}{l}0 . \\
8\end{array}$ & 0 & $\begin{array}{l}0 . \\
2\end{array}$ & 6 & 3 & 156 & 107 & 124.8 & -21.4 & 103.4 \\
\hline 11 & 182 & 2 & 1 & 7 & $\begin{array}{l}0 . \\
2\end{array}$ & $\begin{array}{l}0 . \\
1\end{array}$ & $\begin{array}{l}0 . \\
7\end{array}$ & -5 & $\begin{array}{l}8 . \\
5 \\
\end{array}$ & 16 & 220 & 3.2 & -154 & 150.8 \\
\hline
\end{tabular}

It is seen from Table 1 that subject number one with estimated relative performance index of $W_{1}=10$ if based on equation 9 or 731 if based on equation 26 that adjusts for magnitude and direction and has the highest cholesterol level of 267 is the highest rated in terms of cholesterol level. The lowest rated subject is subject number three ranked 11 with a relative performance index of $W_{i}=-10$ if based on equation 9 or -358 if based on equation 26 that is adjusted for both magnitude and direction and has the smallest cholesterol level of 168. Subject number two with relative performance index of of $W_{i}=0$ (equation 9) or -41.5 (equation 26) has a relative performance index that is higher than those by one-half and less than those by another one-half of the sampled population. Hence this subject's cholesterol level of 193 provides a sample estimate of the sampled population's median cholesterol level.

Now the researcher may wish to determine whether subject number 10 ranked 3, that is at the third quartile and subject number five and eleven ranked 8.5 each and at the first quartile of the distribution of cholesterol levels in the sampled population indices, and hence do not differ in their cholesterol levels. Note that the cholesterol level of subject number ten with performance index of $W_{i}=6$ if based only on equation 9 that does not directly reflect the relative magnitude of the observations is 205 , while the cholesterol level of subject number five or eleven with relative performance index of -5 is 182 . Hence to compare these subject-relativeperformance-indices, we have from Table 1 and equation 17 that

$$
\begin{gathered}
\chi^{2}=\frac{(6-(-5))^{2}}{10\left(0.80+0.20-(0.80-0.20)^{2}\right)+\left(0.20+0.70-(0.20-0.70)^{2}\right)} \\
=\frac{121}{12.9}=9.380
\end{gathered}
$$

$(\mathrm{p}$-value $=0.0000)$, which with 1 degree of freedom is highly statistically significant, indicating a significant difference between the relative performance indices and hence between first quartile and third quartile cholesterol levels of subjects from the population.

If we had on the other hand used instead equation 26 which is adjusted for both direction and magnitudes of the observations we would have that the corresponding relative performance indices for subject numbers 10 and 5 are relatively 103.4 and $-150.8\left(W_{i}(26)\right.$ in table 1$)$. Hence to test the null hypothesis that the first quartile and third quartile relative performance indices are the same, when the population is numeric, we would proceed as follows.

Now the sample estimates of the variances of $W_{10}$ and $W_{5}$ are from equation 25 respectively

And

$$
\operatorname{Var}\left(W_{10}\right)=0.16(5869+3742)=0.16(9611)=1537.76
$$

Hence from equation 16 and 25 the estimated variance of $W_{10}-W_{5}$ is

$$
\operatorname{Var}\left(W_{10}-W_{5}\right)=\operatorname{Var}\left(W_{10}\right)+\operatorname{Var}\left(W_{5}\right)=1537.76+34.725=1572.485
$$

Therefore to test the null hypothesis of the equality of the two relative performance indices in the sampled population we have from equation 17 the test statistic 


$$
\chi^{2}=\frac{(103.4-(-150.8))^{2}}{1572.485}=\frac{64617.64}{1572.485}=41.093(p-\text { value }>0.0000)
$$

which with 1 degree of freedom is highly statistically significant again indicating that the first quartile and third quartile subject relative performance indices and hence the corresponding cholesterol levels are significantly different.

Note however that the relative sizes of the chi-square values obtained suggest that the generalized approach based on equation 1 that reflects only the direction but not the relative magnitudes of the observations (cholesterol levels) is likely to lead to an acceptance of a false null hypothesis (Type II error) more frequently and hence is likely to be less powerful than the modified method based on the equation 21 that reflects both the direction and relative magnitudes of the observations. Hence when the observations being analyzed are numeric measurements the modified method of analysis based on equation 221 rather than equation 1 should be adopted. Finally as earlier noted, the proposed method still enables the assignment to each subject exactly the same rank whether the rank is based on the more general subject specific relative performance indices of equation 9 $\left(W_{i}(9)\right)$ obtained using equation 1 , the subject relative performance indices of equation $26\left(W_{i}(26)\right)$ obtained using equation 21 modified when the observations are numeric measurements, or when the observations themselves are merely assigned ranks in the usual way. However it is relatively much easier and quicker with the proposed method, unlike the usual method of rank-ordering observations to estimate the median and the quartile cholesterol levels of the sampled population of school teachers and possibly other tiles if desired.

\section{Summary and Conclusion}

We have in this paper developed and presented a statistical measure here termed Modified Subjects Specific Index of Relative Performance or Modified Subject Specific Relative Performance Index that would enable the rank-ordering of subjects drawn from a population according to their relative performance or scores in a contest, test or condition to guide preferential selection of subjects or subsets of subjects for any remedial actions that may be desired.

Methods that are preferably appropriate for use when the sampled population is information or measurements on only the ordinal scale as well as when the observation s are numerical measurements are provided.

The proposed method easily enables one using the subject specific relative performance indices to estimate the median and other tiles of the population of interest.

Test statistics based on the proposed indices have also been developed to test any hypothesis concerning patterns of relationships between scores by subjects and also concerning the percentiles of the population.

The proposed methods were illustrated with some sample data. The method, modified to adjust for both relative magnitudes and direction of the observation, was as expected found to be more powerful than the ordinal measurements-based method that reflects only the direction but not the magnitude of the observations.

\section{References}

[1]. Arua et al (1997): Fundamentals of Statistics for Higher Education. Fijac Academic Press, Nsukka, Nigeria.

[2]. Gibbons, J. D. and (1971): Nonparametric Statistical Inference. Mercel Dekker, Inc. New York.

[3]. Oyeka, I. C. A. (2012):Introduction to Applied Statistics. Nobern Avocacy Publishers, Enugu, Nigeria.

[4]. Oyeka, I. C. A. (2009): Introduction to Applied Statistics. Nobern Avocacy Publishers, Enugu, Nigeria.

[5]. Oyeka, I. C. A. (1996): Introduction to Applied Statistical Methods in the Sciences. Nobern Avocacy Publushers, Enugu, Nigeria. 